\title{
A Joint Optimization Model of $(s, S)$ Inventory and Supply Strategy Using an Improved PSO-Based Algorithm
}

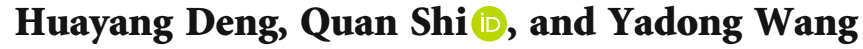 \\ Army Engineering University, Shijiazhuang Campus, Shijiazhuang, Hebei 050003, China \\ Correspondence should be addressed to Quan Shi; anshi2you@163.com
}

Received 9 July 2021; Revised 10 August 2021; Accepted 12 August 2021; Published 31 August 2021

Academic Editor: Thippa Reddy G

Copyright () 2021 Huayang Deng et al. This is an open access article distributed under the Creative Commons Attribution License, which permits unrestricted use, distribution, and reproduction in any medium, provided the original work is properly cited.

\begin{abstract}
This paper mainly discussed the problem of a multiechelon and multiperiod joint policy of inventory and supply network. According to the random lead time and customers' inventory demand, the $(s, S)$ policy was improved. Based on the multiechelon supply network and the improved, the dynasty joint model was built. The supply scheme in every period with the objective of minimum total costs is obtained. Considering the complexity of the model, the improved particle swarm optimization algorithm combining the adaptive inertia weight and grading penalty function is adopted to calculate this model and optimize the spare part problems in various environments.
\end{abstract}

\section{Introduction}

As an important foundation of product maintenance, the research on spare parts in product maintenance is increasingly applied in industrial and military fields. Along with more and more research, it becomes more and more mature.

In the last practice, many researchers have studied many aspects in every field of spare parts. Sherbrooke in the establishment of the aviation spare for maximum availability considered the failure laws of a complex system and established a spare part demand model under complex factors [1]. Hu et al. analyzed the characteristics of various spare part inventory policies and distinguished the best use conditions for different inventory policies [2]. Ghobbar and Friend added dynamic coordination to the study of multistage spare part supply to improve the stability of the spare part supply process and improve system efficiency [3].

With the deepening of the research, the research of spare part work is not limited to one aspect and gradually begins to deepen the research on the whole process of spare part work. Among these, the researchers are more interested in the joint optimization of spare part inventory and supply process. In the study of joint optimization, it is resulting from the flexibility and random factors of maintenance mode, to con- sider multiperiod continuous process than to study spare part strategy for a certain period which is more valuable.

In the research of inventory policy, considering the characteristics of modern inventory, then the $(T, S)$ and $(s, S)$ policies are more in line with the actual inventory management experience [4]. According to the $(T, S)$ policy, the capacity will be replenished to $S$ in the same time interval of time $T$ [5]. As for the $(s, S)$ policy, when the capacity decreases less than $s$, the capacity is replenished whose capacity maximum is $S$. In contrast, the $(s, S)$ policy is more flexible and more complex in the research of inventory policy so that the joint inventory policy is less studied. This paper will make use of the characteristics and advantages of the $(s, S)$ policy in joint optimization to carry out joint optimization research [6].

In the project of joint optimization of inventory and supply, researchers focus on balancing transportation costs and breakdown losses caused by insufficient inventory based on satisfying demand and then determining the supply lead time, to achieve the purpose of maximizing benefits. In many previous works of literatures, fixed supply lead time is used to calculate, but in contrast to practical experience, this assumption is impractical, so much literature began to consider details of this aspect [7]. Reference [6] shows that the supply lead time set is an empirical formula, which is assumed to satisfy Poisson 
distribution in the literature, but the lead time will change dynamically according to the influence of realistic objective, subjective, or random factors. Therefore, the lead time can still be further researched. The joint optimization in this paper can calculate the specific delivery time of each customer by optimizing the supply distribution process. The system optimizes the supply distribution process by adjusting the quantity of spare parts transporting between different nodes. So, the counts of the spare parts transporting between different nodes are regarded as the decision parameters and the optimization factors. The system can reduce the cost while ensuring the inventory consumption throughout the supply cycle as far as possible by adjusting these parameters.

The problem discussed in this paper is a multiechelon supply network optimization problem. This paper used an improved optimization algorithm to solve the problem. An adaptive particle swarm optimization (PSO) algorithm is proposed in this literature [8]. Based on the optimization framework of the traditional PSO algorithm, the improved algorithm can detect and respond to the changes in the optimization environment. Otherwise, we jointed the new parameters, such as the inertia weight and penalty function. The global exploration and local development ability of the algorithm is adjusted in time to improve the efficiency of the algorithm and adopt an adaptive neighborhood search policy when the environment changes.

The rest of this paper is arranged as follows: Section 2 outlines spare part supply, inventory policy, and joint optimization. Section 3 provides a multiperiod spare part supply optimization model based on product characteristics and applying the $(s, S)$ policy. Section 4 introduces the proposed improved PSO algorithm. Section 5 gave a numerical case to analyze the corresponding results. Section 6 combined numerical examples to analyze the sensitivity of the model and compare it with the traditional policy. Conclusions and future work were given in Section 7.

\section{Literature Review}

2.1. Supply Network Optimization. Many researchers study supply networks for a long time. The main research objectives are two aspects. The first one is the supply cycle. Most of research tend to study the supply of spare parts in a single echelon, while multistage supply should be studied in joint optimization. Sherbrooke [9] firstly builds the metric model by multiperiod resupply process. Vaughan studied the multiperiod process of supply to build the ordering policy of spare parts according to random failure possibility [10]. The research on the supply cycle is developed to multiple periods.

Another one is the supply structure. Cachon [11] built the two-echelon supply network. Kennedy et al. [12] research on multiechelon supply process. The research on the supply network structure began to develop from single-echelon to two-echelon and multiechelon.

2.2. Inventory Policy. The inventory strategy, aiming at the optimization goal, can be divided into two main aspects. On the one hand, the periodic inventory strategy, which is mainly represented by the $(T, S)$ and $(T, Q)$ inventory strategy, regards the inventory time as the optimization object. This kind of inventory strategy complements the inventory at the specified time node which is a difference by one period $T$ to replenish the inventory capacity to $S[13,14]$. On the other hand, the other inventory strategy is mainly represented by the $(s, S)$ and $(s, Q)$ inventory strategy. According to the $(s, S)$ and $(s, Q)$ inventory strategy, when the capacity level is equal to or less than $s$, the system will resupply the spare parts to increase inventory up to $S$ or resupply the stable quantity $Q$ of spare parts $[6,15,16]$.

However, when analyzing the inventory strategy of the second kind, the inventory cannot be monitored in real-time under the actual situation, so the interval time of monitoring is still considered in the research process. In the process of optimization, more researchers' points focused on the study of time, so $(s, S)$ and $(s, Q)$ which stand for the second kind of inventory strategy are developed into $(s, S, t)$ and $(s, Q, t)$ inventory strategy $[17,18]$. What is more, the maximum inventory in different nodes can be different.

2.3. Joint Optimization. For the research of joint strategy optimization, more researchers focus on the joint optimization of maintenance and inventory policy, and there are relatively few joint optimization studies on inventory and supply policy.

Federgruen and Zipkin [19] firstly began to consider the joint of inventory and supply. In the present, the research of inventory and supply joint model mostly starts from two aspects. On the one hand, Spanjers et al. [16] used two echelons in the structure of the joint model. Then, Aharon and Boaz studied establishing multiechelon and multiperiod joint models [17]. Furthermore, the joint model of decentralization is established by Aggarwal and Moinzadeh [18].

On the other hand, many researchers begin to study joint optimization through fixed-length check inventory strategy, such as $(T, S)$ and $(T, Q)[14,20]$. However, with the development of joint policy, the complexity of joint optimization is getting deeper and wider. This kind of fixedlength check strategy is not suitable for the developed joint policy. So, some researchers change to another kind of inventory strategy, such as $(s, S)$ and $(s, Q)[10,21]$.

2.4. Solution Algorithm. There are many algorithms that emerged endlessly in the field of algorithm research. The new intelligent algorithm which combined the advantages of different algorithms has also been developed deeply [21-23].

Whether it is a traditional algorithm or a new intelligent algorithm, there are mainly two types, namely, heuristic and metaheuristic [24]. Among most supply models, particle swarm optimization (PSO) is mostly used. The PSO algorithm is a parallel algorithm, which makes use of the advantages of parallel computing of current processors efficiently and greatly improves the efficiency of optimization. Kennedy et al. [12] firstly adopted this algorithm. However, considering the diversity and complexity of the current supply model, the traditional PSO algorithm solves this kind of model problem for a long time and cannot obtain the result even. In order to improve this, many researchers begin to work. One hand is that Clerc and Kennedy [25] adopted the contraction factor 
into the algorithm structure. Leong and Yen [26] adjust the inertia weight according to the particles' positions to speed up the convergence rate. On the other hand, Mezura-Montes and Coello Coello [27] improved the pental function in the algorithm to improve the optimization ability.

The improved algorithm in this paper combines the nonlinear dynamic inertia weight and the penalty factor of dynamic correction used to detect the global optimum. Adjusting the convergence weight of particles in global and local optimization adapts to the multiperiod iteration of the model. It is suitable for solving the multiperiod continuous optimization problem.

\section{Modelling}

3.1. Problem Description Assumptions. The three-echelon spare part supply network consists of supply centers, distribution points, and customers, and the organizational structure is shown in Figure 1. Spare parts are sent from the first-echelon supply centers to the second-echelon reloading points and then from the reloading points to the thirdechelon customers (the third-echelon customers include the used machines and the storage storehouses). Spare part transportation at all echelons does not affect the consumption of spare parts at the third echelon. The used machines at the third echelon are maintained by replacement, and the failure rate of spare parts is determined. The storage storehouses at the third echelon adopted the $(s, S)$ policy [28].

Because the demand for spare parts is multiperiod and the demand is intermittent, the purpose of the model is to minimize the total cost under the previous condition of a certain support rate. By adjusting the supply time of each period, the number of spare part supplies in each period is optimized and the optimal allocation scheme for each period is found.

The plan formulation process is as follows:

First of all, according to the life distribution of parts, the consumption of spare parts in time intervals can be calculated. Then, according to the requirements of the selected inventory policy, calculating the spare part demand is possible in the corresponding periods. Secondly, aiming at the minimum cost, the spare part supply network is constructed, supplemented by the corresponding constraints (node distance, capacity, transportation cost, transportation capacity, and others). According to the capacity level of each storage storehouse to supply at the third echelon, the conditions conclude the supply level of the system; above all, the optimal spare part supply policy will be obtained.

Because the demand for spare parts is multiperiod and the demand is intermittent, the purpose of the model is to minimize the total cost under the previous condition of a certain support rate. By adjusting the supply time of each period, the number of spare part supplies in each period is optimized and the optimal allocation scheme for each period is found.

(1) Assumptions and conditions

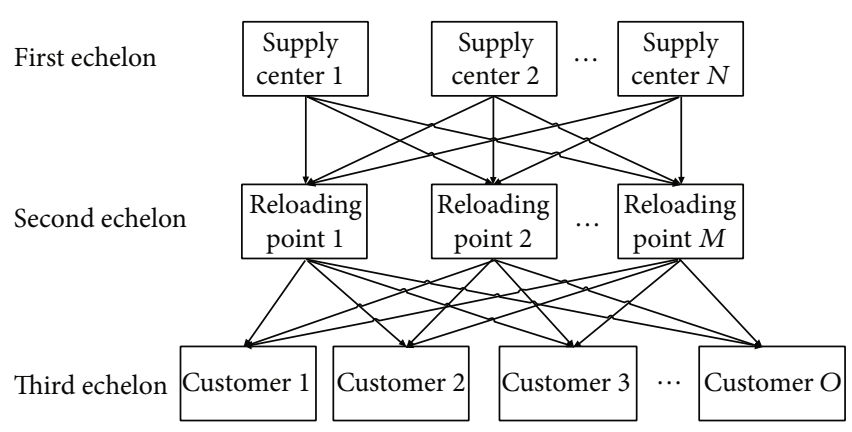

FIgURE 1: Three-echelon supply network.

(a) The capacity of supply centers at the first echelon is unlimited

(b) The second-echelon reloading points only carry on the spare part transshipment and do not store up

(c) This joint model only considers one key kind of parts

(d) Customers at the third echelon have the same importance degree

(2) Notions

$i=1,2,3 \cdots N$ : index of supply centers at first echelon.

$j=1,2,3 \cdots M$ : index of reloading points at second echelon.

$k=1,2,3 \cdots O$ : index of customers at the third echelon.

$\tau=1,2,3 \cdots \psi$ : index of supply periods.

$n_{k}$ : the quantity of customer $k$ 's machines at the third echelon.

$t 0^{\tau}$ : arrival time of spare part resupply to a customer in period $\tau$.

$S_{k}$ : the maximum inventory of customer $k$.

$s_{k}$ : the inventory node of customer $k$.

$t^{\tau}$ : lead time of resupply in period $\tau$.

$T k^{\tau}$ : the supply interval between period $\tau-1$ and period $\tau$ for customer $k$ theoretically.

$T k^{\tau^{\prime}}$ : the time from arriving at customer $k$ 's storage storehouse in the previous period to it in period $\tau$ theoretically, as follows:

$$
T^{k \tau \prime}=t^{k \tau-1}+T^{k \tau}
$$

$T^{\tau}$ : the transporting time of system in period $\tau$.

$h(f)$ : failure probability density function of the equipment resulting from the failure of the spare parts.

$F(h)$ : failure cumulative distribution function of the equipment resulting from the failure of the spare parts.

$p_{k}$ : supply support degree of the customer $k$ at third echelon.

$N_{k}^{T k \tau}$ : consumption of customer $k$ 's spare parts in the supply interval of period $\tau$. 
$N_{k}{ }^{t k \tau}$ : consumption of customer $k^{\prime}$ s spare parts in the lead time of period $\tau$.

$N^{k \tau}$ : consumption of customer $k$ 's spare parts in period $\tau$

$C_{i j}^{p}$ : unit transport cost from supply center $i$ to reloading point $j$.

$C_{j k}{ }^{p}$ : unit transport cost from reloading point $j$ and customer $k$.

$C_{p}:$ total transport cost.

$C_{k}{ }^{v}$ : unit inventory cost of customer $k$ at third echelon.

$C_{k}{ }^{o}$ : ordering cost of customer $k$ at third echelon.

$C_{\tau}^{l}$ : total delay loss by no spare part.

$C_{k}^{l}$ : delay loss for customer $k$ at third echelon by no spare part.

$d_{k}^{\tau}$ : the quantity of resupply spare parts to customer $k$ at third echelon in period $\tau$.

$T_{i j}{ }^{p}$ : the time from supply center $i$ at first echelon to reloading point $j$ at second echelon.

$T_{j k}{ }^{p}$ : the time from reloading point $j$ at second echelon to reloading point $k$ at second echelon.

$w_{p}$ : weight of unit spare part.

$w_{c}$ : limited weight of unit transport vehicle.

$b_{N M}{ }^{c \tau}$ : the count of transport vehicle from $N$ supply centers to $M$ reloading points in period $\tau$.

$b_{M O}{ }^{c \tau}$ : the count of transport vehicle from $M$ reloading points to $O$ customers in period $\tau$.

(3) Decision variable and notations

$X_{i j}^{\tau}$ : the count of the spare parts transporting from supply center $i$ at first echelon to reloading point $j$ at second echelon in period $\tau$.

$X_{j k}^{\tau}$ : the count of the spare parts transporting from reloading point $j$ at second echelon to customer $k$ at third echelon in period $\tau$.

3.2. Calculation of Spare Part Requirements. First of all, according to the principle of demand traction supply and the life distribution of spare parts, within the limited time range of each period, the replacement probability of spare parts (spare part consumption is $s$ ) is as follows:

$$
P_{x}(s)=F^{s}(h)-F^{s+1}(h),
$$

where $F^{s}(h)$ represents $F(h)$ 's s-fold convolution [29]. The formula of supply support degree is as follows:

$$
P=\sum_{s=0}^{N} P_{x}(s)=\sum_{s=0}^{N}\left[F^{s}(h)-F^{s+1}(h)\right]
$$

At the same time, the consumption formula of parts with different life distributions is given as follows:

(a) Assuming that the life of the part is exponentially distributed from the failure rate $\lambda$, the consumption is as follows:

$$
\lambda h+Z_{p} \sqrt{\lambda h}
$$

where $Z_{p}$ is the quantile of the standard normal distribution.

(b) Assuming that the life of the component follows the normal distribution of mean $\mu$ and standard deviation $\sigma$, the consumption is as follows:

$$
\frac{h}{\mu}+Z_{p} \sqrt{\frac{\sigma^{2} h}{\mu^{3}}}
$$

(c) Assuming that the life of the component follows the Weibull distribution with shape parameter $\alpha$, scale parameter $\beta$, and position parameter $\gamma=0$, the expectation of consumption is as follows:

$$
\left[\frac{Z_{p} \Phi}{2}+\sqrt{\left(\frac{Z_{p} \Phi}{2}\right)^{2}+\frac{h}{E}}\right]^{2}
$$

where the expectation $E$ and the variance $\Phi$ are as Equations (7) and (8) in the following:

$$
\begin{gathered}
E=\beta \cdot \Gamma\left(1+\frac{1}{\beta}\right), \\
\Phi=\sqrt{\frac{\Gamma(1+2 / \beta)}{\Gamma(1+1 / \beta)^{2}-1}} .
\end{gathered}
$$

Supply decision starts with spare part demand. Spare part resupply in each period $\tau$ shall meet all spare part consumption of the interval time from the former resupply arriving time $t_{0}^{\tau-1}$ in period $\tau-1$ to the current resupply arriving time $t_{0}^{\tau}$ in period $\tau$. According to the definition of $T^{\tau^{\prime}}$, it can be divided into the counts of consumption in supply interval $T^{\tau}$ and lead time $t^{\tau}$.

According to Equation (2), the spare part consumption of customer $k$ in the supply interval $T_{k}^{\tau}$ of the third echelon is as follows:

$N_{k}^{T^{k \tau}}=n_{k} \cdot \inf \left\{N_{k}^{T^{k \tau}} \mid p_{k} \geq \sum_{s=0}^{N_{k}^{T^{k \tau}}}\left[F^{s}\left(T^{k \tau^{\prime}}-t^{\tau-1}\right)-F^{s+1}\left(T^{k \tau^{\prime}}-t^{\tau-1}\right)\right]\right\}$.

Spare part consumption during lead time $t^{\tau}$ at the third echelon is as follows: 


$$
N_{k}^{k^{k \tau}}=n_{k} \cdot \inf \left\{N_{k}^{k^{k \tau}} \mid p_{k} \geq \sum_{s=0}^{N_{k}^{k \tau}}\left[F^{s}\left(t^{\tau}\right)-F^{s+1}\left(t^{\tau}\right)\right]\right\} .
$$

Therefore, according to Equations (9) and (10), the spare part consumption of customer $k$ in the period $\tau$ is as follows:

$$
N_{k}^{\tau}=n_{k} \cdot \inf \left\{N_{k}^{\tau} \mid p_{k} \geq \sum_{s=0}^{N_{k}^{\tau}}\left[F^{s}\left(T^{k \tau}-t^{\tau-1}+t^{\tau}\right)-F^{s+1}\left(T^{k \tau}-t^{\tau-1}+t^{\tau}\right)\right]\right\} .
$$

3.3. The Joint Optimization Model Based on the $(s, S)$ and Supply Policy. In the decisions of spare part inventory and supply, the model may involve the cost of transportation, inventory costs, ordering costs, downtime loss, and so on.

Due to equipment failure and the reduction of spare part inventory, it is necessary to resupply in advance to ensure the spare parts during maintenance. Otherwise, the huge cost of downtime brings a huge burden to customers.

The goal of the model is to reduce the equipment downtime loss and inventory cost as far as possible and to make the cost of the whole spare part inventory-supply process lowest under a certain equipment availability. In the discussion of this model, the functional model of spare parts is not discussed.

According to the characteristics of the $(s, S)$ policy, the cost of this model includes transportation cost, inventory cost, order cost, and downtime loss. The objective function is as follows:

$$
\min C_{\tau}=C_{\tau}^{p}+\sum_{k}^{O} C_{k}^{v}\left(\sum_{j}^{M} X_{j k}^{\tau}\right)+\sum_{k}^{O} C_{k}^{o}+C_{\tau}^{l} .
$$

Among them, the first item is the transportation cost, the second item is the inventory cost, the third item is the order cost, and the fourth item is the breakdown loss of machines.

According to the $(s, S)$ policy, the consumption of spare parts during the period $\tau$ should be equal to the difference between $S_{k}$ and $s_{k}$, as follows:

$$
N_{k}^{T_{k \tau}}=S_{k}-s_{k} .
$$

Combination with Equations (9) and (13) can be expressed as follows:

$$
n_{k} \cdot \inf \left\{N_{k}^{\tau} \mid p_{k} \geq \sum_{s=0}^{N_{k}^{\tau}}\left[F^{s}\left(T^{k \tau}-t^{\tau-1}+t^{\tau}\right)-F^{s+1}\left(T^{k \tau}-t^{\tau-1}+t^{\tau}\right)\right]\right\}=S_{k}-s_{k} .
$$

According to Equation (14), the system supply time of the third echelon in the period $\tau$ can be obtained, but in the actual supply, the model generally adopts the unified supply, and the supply time should be determined by the supply level of inventory capacity in the third echelon. The actual supply time should be the maximum theoretical time in the third echelon:

$$
T^{\tau}=\max \left(T^{k \tau}\right) .
$$

Therefore, Equation (14) can be adjusted to

$$
n_{k} \cdot \inf \left\{N_{k}^{\tau} \mid p_{k} \geq \sum_{s=0}^{N_{k}^{\tau}}\left[F^{s}\left(T^{\tau}-t^{\tau-1}+t^{\tau}\right)-F^{s+1}\left(T^{\tau}-t^{\tau-1}+t^{\tau}\right)\right]\right\}=S_{k}-s_{k}{ }^{\prime} .
$$

From Equation (16), the resupply nodes in customers' inventories in fact is determined by which inventory reaching the supply point at the latest.

The resupply quantity of spare parts during the period $\tau$ should bring the inventory of each cunstomer back to the maximum. When there are spare parts in store, the quantity should be the difference between the maximum inventory and the remaining inventory. When the spare parts are used up, the quantity should be equal to the maximum. It should be expressed as follows:

$$
d_{k}^{\tau}=\sum_{j}^{M} X_{j k}^{\tau}=\min \left(S_{k}, N_{k}^{\tau}\right) .
$$

Secondly, since the reloading points have no inventory capacity, the output of spare parts should be equal to the input of the reloading points, as follows:

$$
\sum_{i}^{N} X_{i j}^{\tau}=\sum_{k}^{O} X_{j k}^{\tau}
$$

Furthermore, the quantity of transport is limited and is not higher than the maximum transfer capacity of each reloading point, as follows:

$$
\sum_{i}^{N} X_{i j}^{\tau} \leq U_{j}
$$

At the same time, in the resupply process of spare parts, the part of downtime loss should be considered, as follows:

$$
C_{\tau}^{l}=\sum_{k}^{O} C_{k}^{l} n_{k}\left(-\min \left(s_{k}-N_{k}^{t^{k \tau}}, 0\right)\right) .
$$

When the remaining spare parts in inventory are sufficient to meet the spare part consumption in the lead time $t^{\tau}$, there is no downtime loss, as follows:

$$
s_{k}-N_{k}^{t_{k \tau}} \geq 0
$$

When the remaining spare parts cannot do it, it is necessary to bear the downtime loss of machines that cannot replace spare parts, as in Equations (22) and (23) in the following:

$$
s_{k}-N_{k}^{t_{k \tau}}<0
$$




$$
C_{\tau}^{l}=\sum_{k}^{O} C_{k}^{l} n_{k}\left(s_{k}-N_{k}^{t_{k \tau}}\right)
$$

In the course of transport, taking into account the transport limit of vehicles, the total weight transported by a single means of transport shall not exceed the limit specified by the means of transport, as in Equations (24) and (25) in the following:

$$
\begin{gathered}
b_{N M}^{c \tau}=\sum_{i}^{N} \sum_{j}^{M}\left[X_{i j}^{\tau} \times \frac{w_{c}}{w_{p}}\right], \\
b_{M O}^{c \tau}=\sum_{j}^{M} \sum_{k}^{O}\left[X_{j k}^{\tau} \times \frac{w_{c}}{w_{p}}\right],
\end{gathered}
$$

where the Gaussian function is used in the above formulas, which is rounding up. When the resupply weight exceeds the limit weight for a vehicle, an additional vehicle is needed.

The transport cost is related to the distance of transport carrying out transport, as follows:

$$
\begin{aligned}
C_{\tau}^{p}= & b_{N M}^{c \tau} \times C_{i j}^{p}+b_{M O}^{c \tau} \times C_{j k}^{p}=\sum_{i}^{N} \sum_{j}^{M}\left(\left[X_{i j}^{\tau} \times \frac{w_{c}}{w_{p}}\right] \times C_{i j}^{p}\right) \\
& +\sum_{j}^{M} \sum_{k}^{O}\left(\left[X_{j k}^{\tau} \times \frac{w_{c}}{w_{p}}\right] \times C_{j k}^{p}\right) .
\end{aligned}
$$

In this model, it is one of the previous conditions that spare parts can start to be delivered to customers only after all spare parts arrive at the reloading points. For different customers, the delivery time between supply centers and reloading points is the same, but the delivery time between reloading points and customers is determined by the time of the spare parts arriving at the customers. Therefore, the customer's arriving time is different from each other, and the span of the transportation time is related to the quantity of spare parts transported. follows:

In period $\tau$, the lead time $t^{\tau}$ for the entire system is as

$$
t^{\tau}=\max \left(X_{i j}^{\tau} T_{i j}^{p}\right)+\max \left(X_{j k}^{\tau} T_{j k}^{p}\right)
$$

The lead time is determined by the amount of spare parts transported between nodes and transportation time. The system would not start the next resupply until the current supply ends. So, the lead time should be equal to the maximum resupply time between nodes.

At the same time, as shown in Equation (28) in the following:

$$
t^{0}=0
$$

where the beginning of the system needs not a resupply.
There is a one more thing that the variable is the natural number and positive number, as follows:

$$
X_{i j}^{\tau}, X_{j k}^{\tau} \in N^{+}
$$

Composed with the previous formulas, the multiperiod joint model of inventory and supply is as follows:

$$
\begin{aligned}
& \min C_{\tau}=\sum_{i}^{N} \sum_{j}^{M}\left(\left[X_{i j}^{\tau} \times \frac{w_{c}}{w_{p}}\right] \times C_{i j}^{p}\right)+\sum_{j}^{M} \sum_{k}^{O}\left(\left[X_{j k}^{\tau} \times \frac{w_{c}}{w_{p}}\right] \times C_{j k}^{p}\right)+\sum_{k}^{o} C_{k}^{v}\left(\sum_{j}^{M} X_{j k}^{\tau}\right)+\sum_{k}^{O} C_{k}^{o} \\
& \text { s.t. } X_{i j}^{\tau}, X_{j k}^{\tau} \in N^{+} \text {, } \\
& N_{k}^{\tau}=n_{k} \cdot\left\{N_{k}^{\tau} \mid p_{k} \geq \sum_{s=0}^{N_{k}^{\tau}}\left[F^{s}\left(T^{k \tau}-t^{\tau-1}+t^{\tau}\right)-F^{s+1}\left(T^{k \tau}-t^{\tau-1}+t^{\tau}\right)\right]\right\}, \\
& d_{k}^{\tau}=\sum_{j}^{M} X_{j k}^{\tau}=\min \left(S_{k}, N_{k}^{\tau}\right), \\
& n_{k} \cdot \inf \left\{N_{k}^{\tau} \mid p_{k} \geq \sum_{s=0}^{N_{k}^{\tau}}\left[F^{s}\left(T^{\tau}-t^{\tau-1}+t^{\tau}\right)-F^{s+1}\left(T^{\tau}-t^{\tau-1}+t^{\tau}\right)\right]\right\}=S_{k}-s_{k}{ }^{\prime}, \\
& \sum_{i}^{N} X_{i j}^{\tau} \leq U_{j} \\
& \sum_{i}^{N} X_{i j}^{\tau}=\sum_{k}^{o} X_{j k}^{\tau} \\
& t^{\tau}=\max \left(X_{i j}^{\tau} T_{i j}^{p}\right)+\max \left(X_{j k}^{\tau} T_{j k}^{p}\right), \\
& t^{0}=0, \\
& N_{k}^{t^{\tau}}=n_{k} \cdot \inf \left\{N_{k}^{t^{\tau}} \mid p_{k} \geq \sum_{s=0}^{N_{k}^{t_{\tau}}}\left[F^{s}\left(t^{\tau}\right)-F^{s+1}\left(t^{\tau}\right)\right]\right\}, \\
& i=1,2,3 \cdots N ; j=1,2,3 \cdots M ; k=1,2,3 \cdots O ; \\
& \tau=1,2,3 \cdots \psi \text {, } \\
& X_{i j}^{\tau}, X_{j k}^{\tau} \in N^{+} \text {. }
\end{aligned}
$$

\section{Proposed Algorithm}

For calculating the proposed model, it is used the intelligent algorithm in this paper. Because of the learning ability of them, especially the PSO algorithm, it can make good use of the existing resources to search the optional decision variables fully, so the PSO algorithm is a good method to solve it. So, we decide to adopt the PSO algorithm to solve this problem.

On the other hand, there are many assumptions and conditions established in this article. Because of that, there are many model constraints, and the target environment structure is more complicated. As the number of data increases, the solution of the PSO algorithm is likely to enter the "local selection trap" and thus cannot obtain the global optimal solution. Therefore, in order to solve this problem, this paper used an improved PSO algorithm.

4.1. Traditional PSO Algorithm. Set the potential solution to the optimization problem as a group of particles in space. Suppose there are $N$ particles in a $D$-dimensional search space, and the vector of the $i^{\text {th }}$ particle in the $D$-dimensional space is expressed as Equation (31) [30].

$$
X_{i}=\left(x_{i 1}, x_{i 2}, x_{i 3} \cdots x_{i D}\right), \quad i=1,2,3 \cdots N .
$$

Each particles have an adaptive value (fitness value) 
determined by its location and passing velocity. The "flying" velocity is as follows:

$$
V_{i}=\left(v_{i 1}, v_{i 2}, v_{i 3} \cdots v_{i D}\right), \quad i=1,2,3 \cdots N .
$$

Their location updates are as in Equations (33) and (34) in the following:

$$
v_{i d}(t+1)=w \cdot v_{i d}(t)+c_{1} \cdot r_{1} \cdot\left[p_{i d}-x_{i d}(t)\right]+c_{2} \cdot r_{2} \cdot\left[p_{i d}-x_{i d}(t)\right],
$$

$$
x_{i d}(t+1)=x_{i d}(t)+v_{i d}(t+1) .
$$

Among them, $P_{\text {best }}^{i}$ is the $i^{\text {th }}$ particle that so far obtained the optimal position. The individual extreme point is recorded as follows:

$$
P_{\text {best }}^{i}=\left(p_{i 1}, p_{i 2}, p_{i 3} \cdots p_{i D}\right), \quad i=1,2,3 \cdots N .
$$

$G_{\text {best }}$ is the optimal position that the entire particle swarm has searched so far. The global extreme point is denoted as follows:

$$
G_{\text {best }}=\left(g_{i 1}, g_{i 2}, g_{i 3} \cdots g_{i D}\right), \quad i=1,2,3 \cdots N \text {. }
$$

For inertia weight $w$, it is used to represent the influence of the initial state on the particle motion. The acceleration degree $\left(c_{1}, c_{2}\right)$ and the acceleration weight coefficient $\left(r_{1}, r_{2}\right.$ ), which are composed of the last two terms of Equation (33), represent the influence of the particle's own historical experience data and the collective historical data on the particle motion. Find the position of the optimal fitness value particles, compare and adjust the local and global extremum points. From then on, repeat Equations (33) and (34), update the local and global extremum, and get the optimal extremum of the system [31].

However, the PSO algorithm also has its disadvantages. For an in-depth discussion of it, one of the biggest advantages lies in the application of the algorithm. The adjustment parameters are less, but they directly affect the performance and convergence of the algorithm [32]. For the weight parameter $w$, the traditional PSO algorithm can improve the global search ability and reduce the local search ability of the algorithm. Therefore, many researchers began to put forward many programmers that improved the weight parameter $w$, such as the adaptive weight method, the random weight method, and the linear recursive weight method.

4.2. Adaptive Weight Method. The adaptive weight method mainly has two kinds of optimization directions. Firstly, according to the early convergence degree of particle swarm, and the value of population fitness, the change of inertia weight $w$ is determined, and the population is divided into three subsets. It is known that the velocity of the algorithm's constringency depends on the dispersion degree of particles. Therefore, when the dispersion degree of particles is a little dispersed, the adaptive weight method reveals details that decreasing the value of $w$ does well in speeding up the veloc-

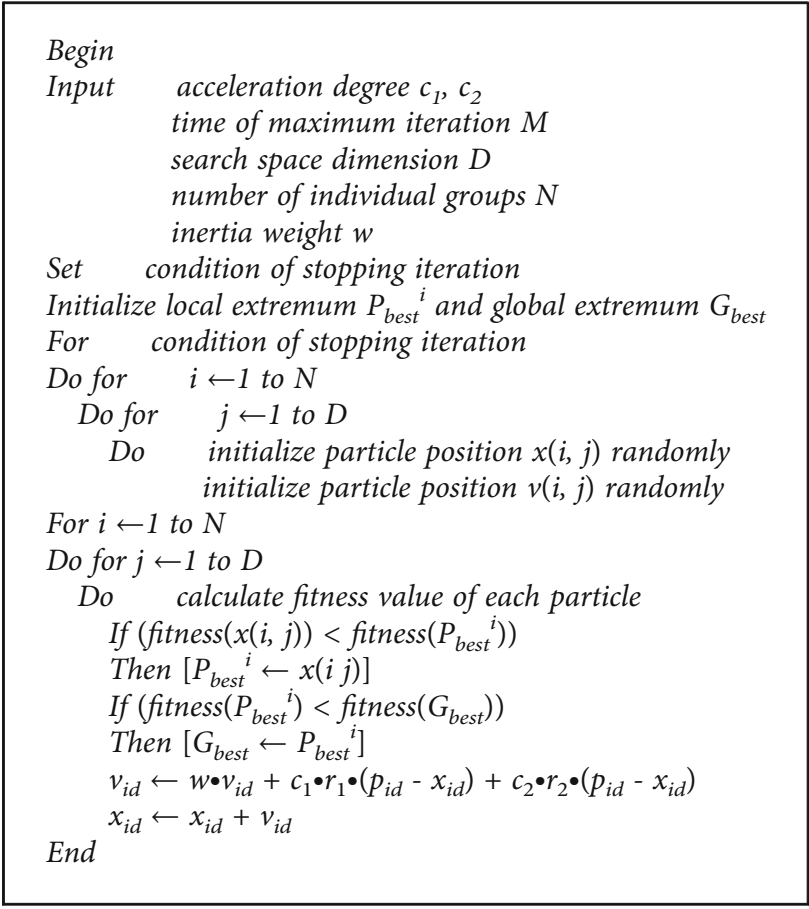

Algorithm 1: Traditional PSO algorithm.

ity of algorithm's constringency. On the other hand, it can raise the value of $w$ to decrease the degree of falling into the "local optimal" trap either [32-34].

Secondly, there is also a method used in this paper to adjust the inertia weight $w$ for the global optimal distance from the current position, as shown in Figure 2. Because the PSO algorithm gets closer to the global optimal in theory through optimization, it decreases $w$ continuously with iterations. On the contrary, it will increase $w$ to strengthen the ability of global research [35].

According to the former discussion, the nonlinear dynamic inertia weight coefficient is determined by the current position. The formula is as follows:

$w= \begin{cases}w_{\min }-\frac{\left(w_{\max }-w_{\max }\right) \times\left(\text { fitness }- \text { fitness }_{\min }\right)}{\text { fitness }_{\text {avg }}-\text { fitness }_{\min }}, & \text { fitness } \leq \text { fitness }_{\text {avg }}, \\ w_{\max }, & \text { fitness }>\text { fitness }_{\text {avg }},\end{cases}$

where fitness represents the current fitness value, fitness ${ }_{\text {min }}$ and fitness ${ }_{\text {avg }}$ represent the minimum and average of the fitness values of all current particles. It can be seen from Equation (37) that when the adaptation value difference of each particle is getting larger, the inertia weight $w$ will be dereasing. When the adaptation value of each particle swarm is getting closer, the inertia weight $w$ will be increasing.

4.3. Fitness Function. In order to deepen the fluence of the constraints in the optimization, the penalty function is introduced into the adaptive function. As a common method to deal with constraints, the penalty function method can transform the constraint optimization problem into an unconstrained 


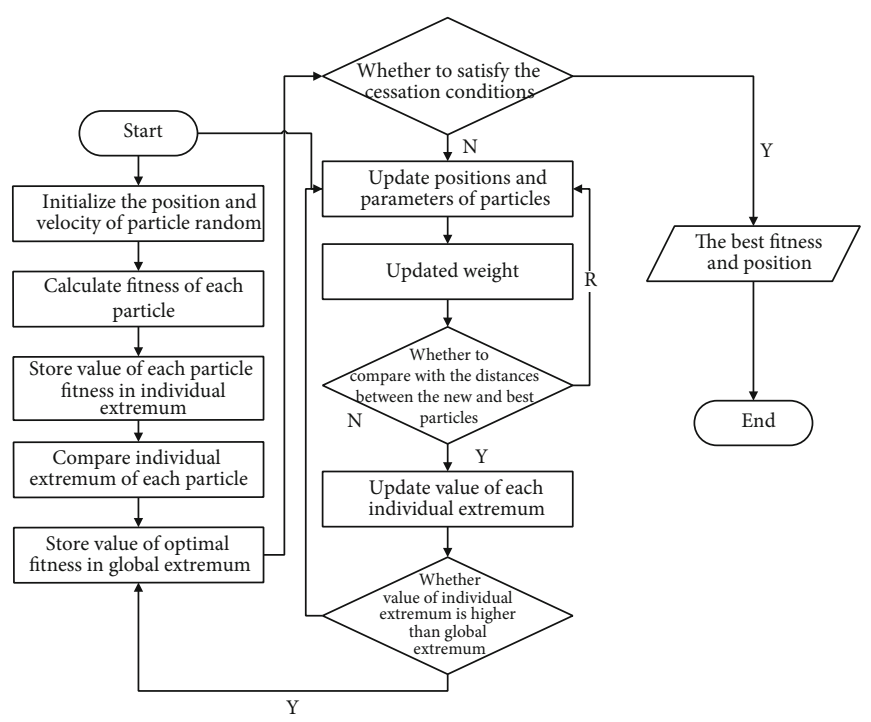

Figure 2: Adjusting the inertial parameter flow at the best global distance.

optimization problem. The punishment function method is mainly divided into an internal punishment method and external punishment method. Set the appropriate parameters according to the constraint conditions and establish the penalty function.

The adaptive function is composed of three parts, which conclude the set variables, the optimized objective function, and the variable constraint condition. The general formula is as follows:

$$
L[x(i, j), k]=\text { fitness }[x(i, j)]+\sigma \cdot P[x(i, j)],
$$

where fitness $[x(i, j)]$ is the objective function, $\sigma$ is the penalty factor, and $P[x(i, j)]$ is the limit function.

A limit function is a set of $n(n=1,2,3 \cdots N)$ limiting conditions, as follows:

$$
P[x(i, j)]=\sum_{n=1}^{N} G_{n}[x(i, j)]
$$

Among them, $G_{n}[x(i, j)]$ is the function of the corresponding constraint condition, which is affected by the constraint function, as follows:

$P[x(i, j)]=\sum_{n=1}^{l} \max \left\{0, g_{n}[x(i, j)]\right\}+\sum_{n=1}^{m} \max \left\{0,\left|h_{n}[x(i, j)]\right|-\delta\right\}$,

where $m$ is the quantity of constraints and $\delta$ is the tolerance value of the equality constraint. It is the unequal constraint, when the condition is as follows:

$$
g_{n}[x(i, j)] \leq 0
$$

It is the equivalent constraint, when the condition is as follows:

$$
h_{n}[x(i, j)]=0,(\delta \longrightarrow 0) .
$$

In most algorithms, a fixed penalty value method is generally used. With the iterations of the particle swarm algorithm, the parameters of the group will change. A dynamic correction method whose penalty value changes with the constraint value is shown in Reference [34]. The general formula of the penalty function is as follows:

$$
L[x(i, j), k]=\text { fitness }[x(i, j)]+h(k) \cdot P[x(i, j)] .
$$

Among them, the penalty factor $h(k)$ is obtained with the number of iteration $k$ increases, and the limit function $P[x(i, j)]$ is improved to satisfy the requirements of multistage iterative changes, as follows:

$$
P[x(i, j)]=\sum_{n=1}^{m} \theta\left\{q_{n}[x(i, j)]\right\} \cdot q_{n}[x(i, j)]^{\gamma\left\{q_{n}[x(i, j)]\right\}},
$$

where $q_{n}[x(i, j)], \theta\left\{q_{n}[x(i, j)]\right\}$, and $\gamma\left\{q_{n}[x(i, j)]\right\}$ are the corresponding series of violation constraint function, multiiteration distribution function, and penalty function.

The search space of the constrained optimization problem is composed of feasible points and infeasible points. The feasible points satisfy all the constraints, and the infeasible points violate at least one constraint. Penalty function technology solves the constrained optimization problem through penalty constraints. If the penalty value of penalty function is too high, the optimization algorithm is easy to converge to the local minimum solution. If the penalty value is too low, it is difficult to find a feasible optimization solution. The penalty function is divided into fixed penalty value and dynamic correction of penalty value. The penalty function depends on the constraint condition. The optimization result obtained by the dynamic modification of the penalty value with the change of the constraint value is better than that of the fixed penalty value. 


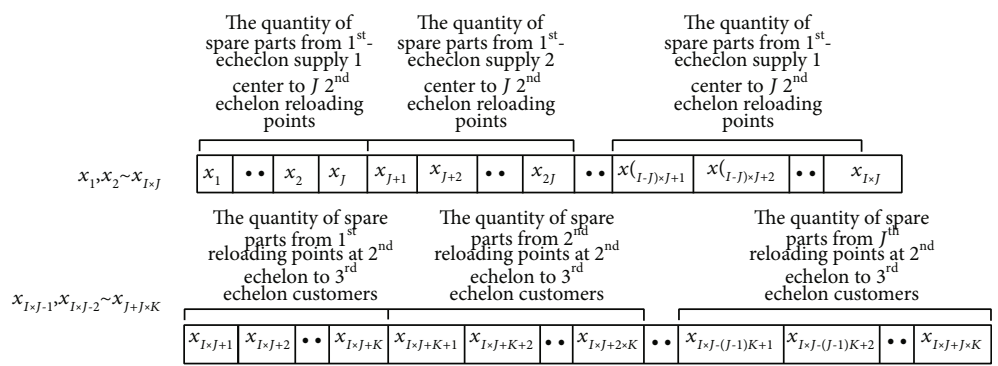

FIgURE 3: Schematic diagram of particle code.

Above the former discussion, the rules of corresponding parameter satisfied are as the following:

(1) When

$q_{n}[x(i, j)]<1, \gamma\left\{q_{n}[x(i, j)]\right\}=1, \quad n=1,2,3 \cdots m$

(2) When

$q_{n}[x(i, j)]<1, \gamma\left\{q_{n}[x(i, j)]\right\}=1, \quad n=1,2,3 \cdots m$

(3) When

$q_{n}[x(i, j)]<1, \gamma\left\{q_{n}[x(i, j)]\right\}=1, \quad n=1,2,3 \cdots m$

(4) When

$0.001 \leq q_{n}[x(i, j)]<0.1, \theta\left\{q_{n}[x(i, j)]\right\}=20, \quad n=1,2,3 \cdots m$

(5) When

$0.1 \leq q_{n}[x(i, j)]<1, \theta\left\{q_{n}[x(i, j)]\right\}=100, \quad n=1,2,3 \cdots m$

(6) When

$q_{n}[x(i, j)] \geq 1, \theta\left\{q_{n}[x(i, j)]\right\}=300, \quad n=1,2,3 \cdots m$

It can be seen from the penalty function value corresponding to the function value listed above that for different limit function values, the penalty function is classified. The closer the function value is, the smaller the penalty amount. On the contrary, the farther the value is, the penalty the larger the value of the function, the stronger the convergence efficiency of the improved PSO algorithm mentioned in the article.

4.4. Particle Coding. In this article, an integer coding method is used to index each particle, and the variation of each dimension of each particle represents the number of spare parts transported between different nodes.

As shown in Figure 3, it represents the quantity of the spare parts transferring to every second-echelon reloading point in the first-echelon supply center $i$. Resulting from it, $x_{1} \sim x_{J}$ represent the number of spare parts transferred from the first-echelon supply center 1 to the second-echelon reloading points, $x_{J+1} \sim x_{J \times 2}$ represent the number of spare parts transferred from the first-echelon supply center 2 to the second-echelon reloading points, and so on. While $x_{I \times J+1} \sim x_{I \times J+K}$ are the quantity of transporting from second-echelon reloading point 1 to the storage storehouses of third-echelon customers, $x_{I \times J+K+1} \sim x_{I \times J+K \times 2}$ are the quantity of transporting from second-echelon reloading point 2 to the storage storehouses of third-echelon customers, etc.

4.5. Improved Algorithm Framework. To sum up, this paper combined the inertial weight method and the multistage allocation penalty function method to adjust the global optimal distance from the current position and adopts the improved PSO algorithm. The following is the main process of the improved algorithm:

\section{Case Analysis}

5.1. Case Description. This paper will provide a case to show the actual optimization effect and test the correctness of the established model and algorithm. Data comes from a spare parts supplier in 2019.

At present, a kind of machine has widely used in a certain area, so that there is a demand that 6 customers need to be supplied spare parts to the three reloading points at first echelon by one supply center at first echelon. When the inventory level of spare parts' capacity reaches the set spare part supply level, the customers send out the required supply information. The first-echelon supply center supplies the same. In this case, all customers are factories assuming that six factories use the machine in the area. Considering the complexity of the distance and transportation 


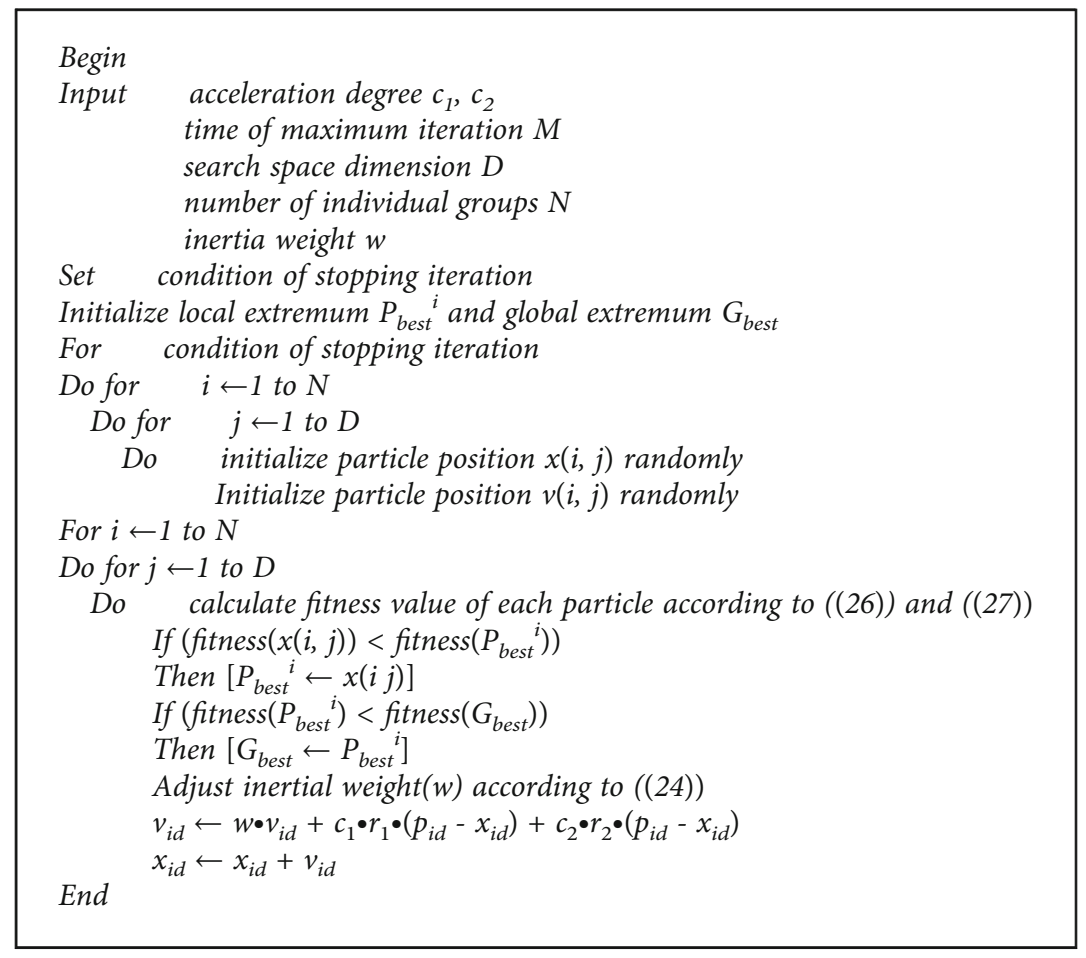

Algorithm 2: Improved PSO algorithm.

TABLE 1: Failure distribution of the discussed part.

\begin{tabular}{lr}
\hline Name & Detail \\
\hline Failure probability $(\lambda)$ & $0.6 \times 10^{-4}$ time/hour \\
Failure distribution function & $F(t)=1-e^{-\lambda t}$ \\
Failure distribution convolutional function & $F^{s}(t)=\sum_{n}^{s-1} \frac{1}{n !}(\lambda t)^{n} e^{-\lambda t}$ \\
\hline
\end{tabular}

conditions, the factories reserve spare parts for this kind of machine, but because of the particularity of the parts using this kind of spare parts, these kinds of parts can only be used in the factory for the maintenance and replacement of the machine. The lifetime distribution of parts is known, as shown in Table 1.

According to the practice, the production environment parameters, such as the number of machines put into production, the number of spare parts stored, the maximum inventory, and the supply support required by production are different. At the same time, due to the influence of regional, production products and policies, various factories pay the cost of ordering, inventory, or breakdown losses caused by spare parts differently. According to statistics, the relevant parameters and costs are shown in Table 2, among which the left five columns are production environment parameters, which are the number of machines put into production in each factory, the supply level, the maximum inventory, and the supply support. The three in the right are listed as economic costs, which are spare part storage cost, order cost, and delay cost.
Transportation costs are one of the most important aspects of the cost; as a result of transport conditions and policies, the cost of transporting spare parts from the firstechelon supply center to the second-echelon reloading points and the second echelon to the storage storehouse of third-echelon factories is different, as shown in Table 3. What is more, the cost of transporting spare parts from the second-echelon reloading point to the third-echelon factory is lower than the cost of transferring spare parts from the first-echelon supply center to the second-echelon reloading point due to the impact of transport conditions and policies. While the reloading points have their own limitations, the maximum transportation volume is set to 100,100 , and 120 .

In spare part supply, another very important parameter is time. A large part of the time in the actual supply process is determined by the volume of spare part transportation. So, the transportation time of unit spare parts in the supply structure is provided in Table 4 in the parameter setting.

In this paper, we divided the whole supply process into five periods, ignoring the transmission time of spare part information. At the same time, the supply process adopted 
TABLE 2: The correlative parameters of third-echelon factories.

\begin{tabular}{|c|c|c|c|c|c|c|c|c|}
\hline & $n_{k}$ & $s_{k}{ }^{\prime}$ & $S_{k}$ & $P_{k}$ & $Z_{p}$ & $C_{k}^{v}$ (unit: $¥$ ) & $C_{k}^{o}$ (unit: $\left.¥\right)$ & $C_{k}^{l}$ (unit: $\left.¥\right)$ \\
\hline Factory 1 & 10 & 20 & 60 & 0.90 & 1.28 & 200 & 2000 & 15000 \\
\hline Factory 2 & 10 & 10 & 60 & 0.99 & 2.33 & 220 & 2000 & 25000 \\
\hline Factory 3 & 8 & 30 & 70 & 0.95 & 1.65 & 200 & 2000 & 20000 \\
\hline Factory 4 & 5 & 25 & 45 & 0.90 & 1.28 & 210 & 2000 & 15000 \\
\hline Factory 5 & 10 & 20 & 50 & 0.80 & 0.84 & 250 & 2000 & 10000 \\
\hline Factory 6 & 12 & 20 & 80 & 0.99 & 2.33 & 225 & 2000 & 10000 \\
\hline
\end{tabular}

TABLE 3: The correlative parameters of third-echelon factories.

\begin{tabular}{lccccccc}
\hline & Supply center & Factory 1 & Factory 2 & Factory 3 & Factory 4 & Factory 5 & Factory 6 \\
\hline Reloading point 1 & 5700 & 600 & 480 & 540 & 480 & 480 & 570 \\
Reloading point 2 & 6000 & 480 & 450 & 510 & 450 & 510 & 630 \\
Reloading point 3 & 4800 & 510 & 330 & 570 & 600 & 510 & 480 \\
\hline
\end{tabular}

TABLe 4: Transportation time of spare parts (unit: km).

\begin{tabular}{lccccccc}
\hline & Supply center & Factory 1 & Factory 2 & Factory 3 & Factory 4 & Factory 5 & Factory 6 \\
\hline Reloading point 1 & 13 & 5 & 2 & 2 & 4 & 3 & 2 \\
Reloading point 2 & 17 & 3 & 6 & 1 & 1 & 2 & 3 \\
Reloading point 3 & 16 & 1 & 1 & 3 & 2 & 2 \\
\hline
\end{tabular}

road transportation which is widely used. The weight of spare parts is 0.5 tons, and the carrying weight of transporting vehicles is limited to 31 tons, without considering the high medium limit of transportation. The best supply scheme is obtained by calculating the above parameters.

Finally, set the PSO algorithm parameter: the maximum number of iterations $M=300$, the number of individual groups $N=200$, and the penalty factor is as follows:

$$
h(k)=k \sqrt{k},
$$

learning factor $c_{1}=1.6962, c_{2}=1.8962$, and the range of inertia weight $\left[w_{\min }, w_{\max }\right]=[0.6,0.8]$.

5.2. Analysis Process. For the joint model in this paper, Equation (18) shows that the transferring quantity of spare parts from first to second is the same as the quantity transferred from first to third echelon. In the calculation of this case, to decrease the decision parameters and programming space, the code schematic can be simplified to encode the secondlevel supply. The simplified code is shown in Figure 4.

The simplified code schematic transforms constraints into logical relationships between decision parameters to speed up the convergence rate. $X_{1} \sim x_{K}$ represent the number of spare parts transported from the second-echelon reloading point 1 to the storage storehouses of thirdechelon customers, $x_{K+1} \sim x_{2 \times K}$ are the number of spare parts transported from the second-echelon reloading point 2 to the storage storehouses of third-echelon customers,

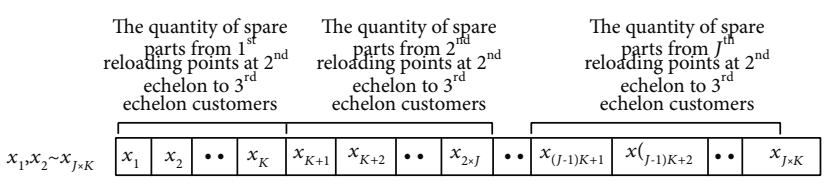

FIGURE 4: Simplified particle code schematic.

etc. What is more, the sum of each group is the number of spare parts transported to the second-echelon reloading points at the corresponding first-echelon supply center, such as the sum of spare parts which includes the number of $x_{K+1} \sim x_{2 \times K}$ which is equal to the number of spare parts transported to the second-echelon reloading points at the first-echelon supply center 1 .

5.3. Result Analysis. According to the improved PSO algorithm proposed in this paper, the corresponding calculation results are obtained in Table 5 .

As shown in Figure 5, they are the change of the objective function, fitness function, and penalty function with the number of iterations.

In Figure 5, the longitudinal axis represents the function value, and the transverse axis represents the iteration period and the number of iterations, where Figure 5(b)) represents the change of the penalty function following the objective function and Figure 5(c)) represents the convergence of the objective function.

It is learning that the adaptive function and objective function tend to converge slowly with the increase of iteration 
TABLE 5: Results of calculation by improved PSO.

\begin{tabular}{lcccccccccccccccccc}
\hline & 1 & 2 & 3 & 4 & 5 & 6 & 7 & 8 & 9 & 10 & 11 & 12 & 13 & 14 & 15 & 16 & 17 & 18 \\
\hline Period 1 & 6 & 4 & 0 & 8 & 17 & 9 & 0 & 8 & 24 & 8 & 12 & 9 & 0 & 12 & 19 & 8 & 16 & 43 \\
Period 2 & 0 & 7 & 23 & 7 & 11 & 10 & 6 & 8 & 24 & 17 & 14 & 0 & 0 & 9 & 12 & 26 & 0 & 7 \\
Period 3 & 0 & 8 & 12 & 10 & 12 & 4 & 0 & 0 & 0 & 20 & 13 & 0 & 0 & 0 & 42 & 9 & 0 & 9 \\
Period 4 & 0 & 5 & 40 & 8 & 12 & 6 & 0 & 0 & 14 & 8 & 6 & 5 & 0 & 44 & 0 & 6 & 15 & 8 \\
Period 5 & 35 & 0 & 15 & 7 & 15 & 5 & 0 & 8 & 0 & 11 & 12 & 1 & 0 & 12 & 0 & 10 & 14 & 8 \\
\hline
\end{tabular}

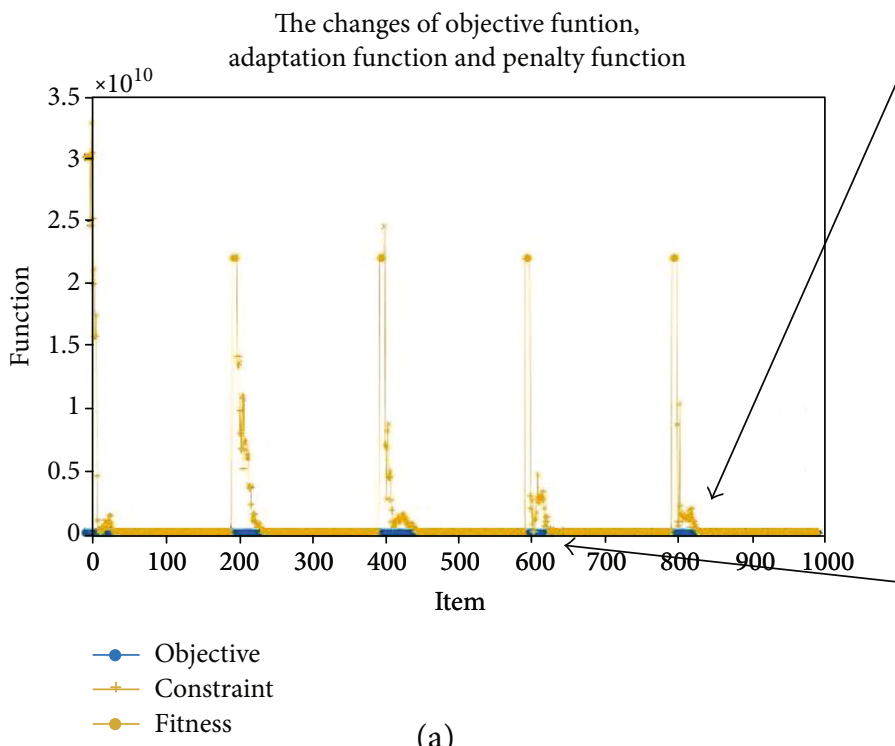

(a)

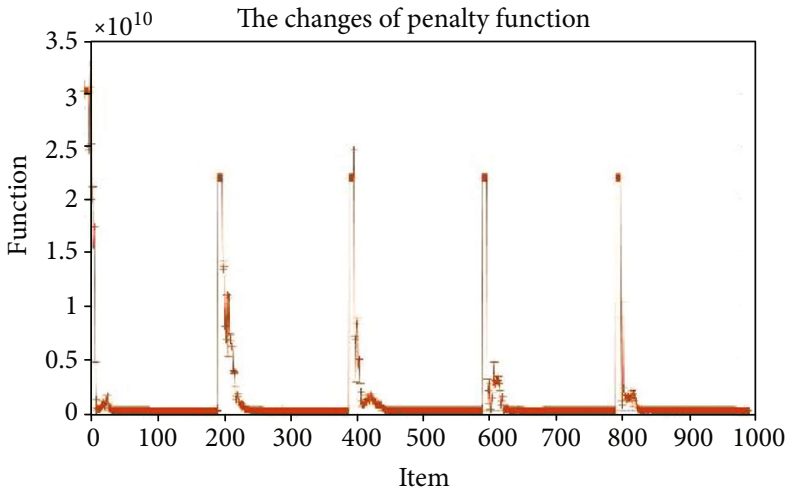

(b)

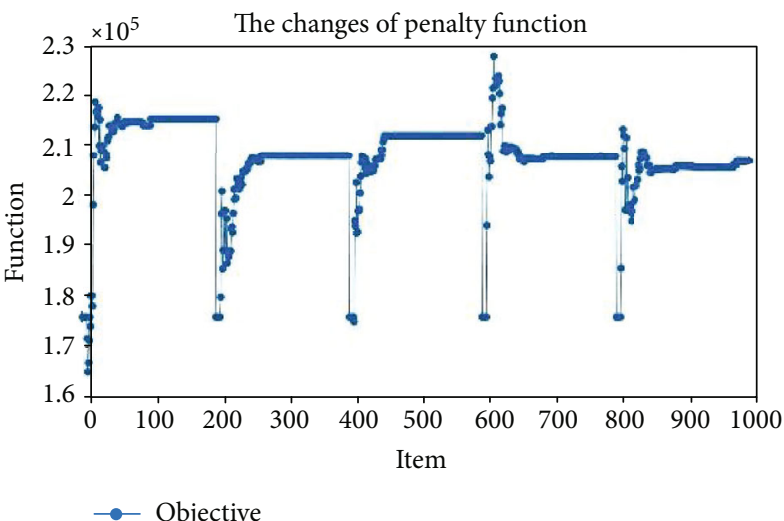

(c)

FIGURE 5: Simplified particle code schematic.

period and times from Figures 5(a) and 5(c)). They tend to be the same at the same time, which shows that the convergence of the results is better, and the convergence of the first stage is slow.

On the other hand, since the adaptive weights used in this paper are closely related to the value of the objective function, the curve of the penalty function in Figure 5(b)) is similar to the objective function. At the same time, the fitting value when all periods are finished tends to 0 , indicating that the result is the minimized optimal solution.

The results of the decision variables are shown in Tables 5 and 6. As can be seen from Table 6, they are shown which are inventory node, the cost of supply at each period, the total cost of the system, and the quantity of spare part delays. Secondly, in Table 5, columns 1-6 are the volume of transport from the second-echelon reloading point 1 to the third-echelon factories, 7-12 are the volume of transport from the secondechelon reloading point 2 to the third-echelon factories, and 13-18 are the volume of transport from the second-echelon reloading point 3 to the third-echelon factories. According to Equation (18) and the former discussion, the volume of transportation from the first-echelon supply center to the secondechelon reloading points can be calculated.

As shown in Table 7, compared to the last three columns included in Table 6, the calculated volume of transport is from the first-echelon supply center to the second-echelon reloading points. Among them, column 19 is the sum of columns 1-6, that is, the transfer volume of the transfer of the 
TABLE 6: Result of the best supply scheme.

\begin{tabular}{lccccc}
\hline & Period 1 & Period 2 & Period 3 & Period 4 & Period 5 \\
\hline Cost (unit: $¥)$ & 137967 & 139404 & 138765 & 138758 & 139907 \\
Total cost (unit: $¥)$ & & & 694801 & & \\
Consumption & {$[30,40,24,15,30,44]$} & {$[30,39,24,17,30,48]$} & {$[30,38,24,15,30,42]$} & {$[30,38,24,14,30,48]$} & {$[30,36,24,15,32,50]$} \\
Level of supply & & & $40,50,34,35,30,44$ & & \\
Breakdown (unit: $¥)$ & {$[0,0,0,0,0,0]$} & {$[0,0,0,0,0,0]$} & {$[0,0,0,0,0,0]$} & {$[0,0,0,0,0,0]$} & {$[0,0,0,0,0,0]$} \\
\hline
\end{tabular}

TABLE 7: Optimized and calculated optimal supply options.

\begin{tabular}{lcccccccccccccccccccccc}
\hline & 1 & 2 & 3 & 4 & 5 & 6 & 7 & 8 & 9 & 10 & 11 & 12 & 13 & 14 & 15 & 16 & 17 & 18 & 19 & 20 & 21 \\
\hline Period 1 & 6 & 4 & 0 & 8 & 17 & 9 & 0 & 8 & 24 & 8 & 12 & 9 & 0 & 12 & 19 & 8 & 16 & 43 & 44 & 61 & 98 \\
Period 2 & 0 & 7 & 23 & 7 & 11 & 10 & 6 & 8 & 24 & 17 & 14 & 0 & 0 & 9 & 12 & 26 & 0 & 7 & 58 & 69 & 54 \\
Period 3 & 0 & 8 & 12 & 10 & 12 & 4 & 0 & 0 & 0 & 20 & 13 & 0 & 0 & 0 & 42 & 9 & 0 & 9 & 46 & 33 & 60 \\
Period 4 & 0 & 5 & 40 & 8 & 12 & 6 & 0 & 0 & 14 & 8 & 6 & 5 & 0 & 44 & 0 & 6 & 15 & 8 & 71 & 33 & 73 \\
Period 5 & 35 & 0 & 15 & 7 & 15 & 5 & 0 & 8 & 0 & 11 & 12 & 1 & 0 & 12 & 0 & 10 & 14 & 8 & 77 & 32 & 44 \\
\hline
\end{tabular}

TABLE 8: Different optimization results of changing the failure degree.

\begin{tabular}{|c|c|c|c|c|}
\hline$\lambda\left(10^{-4}\right)$ & Cost of each period (unit: yuan) & Consumption & Total cost (unit: yuan) & Breakdown loss (unit: yuan) \\
\hline \multirow{5}{*}{0.6} & 137967 & $30,40,24,15,30,44$ & \multirow{5}{*}{694801} & $0,0,0,0,0,0$ \\
\hline & 139404 & $30,39,24,17,30,48$ & & $0,0,0,0,0,0$ \\
\hline & 138765 & $30,38,24,15,30,42$ & & $0,0,0,0,0,0$ \\
\hline & 138758 & $30,38,24,14,30,48$ & & $0,0,0,0,0,0$ \\
\hline & 139907 & $30,36,24,15,32,50$ & & $0,0,0,0,0,0$ \\
\hline \multirow{5}{*}{0.8} & 144112 & $30,40,32,15,30,48$ & \multirow{5}{*}{723862} & $0,0,0,0,0,0$ \\
\hline & 144509 & $30,38,32,15,30,45$ & & $0,0,0,0,0,0$ \\
\hline & 145219 & $30,38,30,15,30,48$ & & $0,0,0,0,0,0$ \\
\hline & 143910 & $30,40,32,18,25,47$ & & $0,0,0,0,0,0$ \\
\hline & 146112 & $35,37,30,15,28,48$ & & $0,0,0,0,0,0$ \\
\hline \multirow{5}{*}{1} & 186998 & $40,60,32,20,30,72$ & \multirow{5}{*}{887221} & $0,0,0,0,0,0$ \\
\hline & 170912 & $40,50,32,20,30,60$ & & $0,0,0,0,0,0$ \\
\hline & 170455 & $40,50,32,20,30,60$ & & $0,0,0,0,0,0$ \\
\hline & 186751 & $40,60,32,20,30,72$ & & $0,0,0,0,0,0$ \\
\hline & 172106 & $40,50,32,20,30,60$ & & $0,0,0,0,0,0$ \\
\hline \multirow{5}{*}{1.2} & 199304 & $40,60,40,20,40,72$ & \multirow{5}{*}{946741} & $0,0,0,0,0,0$ \\
\hline & 185191 & $40,60,32,20,30,72$ & & $0,0,0,0,0,0$ \\
\hline & 188756 & $40,65,32,20,32,72$ & & $0,0,0,0,0,0$ \\
\hline & 185761 & $40,60,32,25,30,58$ & & $0,0,0,0,0,0$ \\
\hline & 187729 & $38,60,35,20,30,66$ & & $0,0,0,0,0,0$ \\
\hline \multirow{5}{*}{1.4} & 3097202 & $40,70,48,20,40,84$ & \multirow{5}{*}{3887792} & $0,0,0,0,0,480000$ \\
\hline & 199658 & $40,60,40,20,40,72$ & & $0,0,0,0,0,0$ \\
\hline & 198548 & $40,65,40,20,35,72$ & & $0,0,0,0,0,0$ \\
\hline & 193576 & $40,60,32,20,40,72$ & & $0,0,0,0,0,0$ \\
\hline & 198807 & $40,60,38,20,40,76$ & & $0,0,0,0,0,0$ \\
\hline
\end{tabular}


TABLE 9: Different optimization results of quantity of inventory to supply.

\begin{tabular}{|c|c|c|c|c|c|}
\hline No. & $\begin{array}{l}\text { Quantity of inventory to } \\
\text { supply }\end{array}$ & $\begin{array}{c}\text { Cost of each period (unit: } \\
\text { yuan) }\end{array}$ & Consumption & $\begin{array}{l}\text { Total cost (unit: } \\
\text { yuan) }\end{array}$ & $\begin{array}{c}\text { Breakdown loss (unit: } \\
\text { yuan) }\end{array}$ \\
\hline 1 & $\begin{array}{l}60 \\
80 \\
50 \\
45 \\
50 \\
80\end{array}$ & $\begin{array}{l}137967 \\
139404 \\
138765 \\
138758 \\
139907\end{array}$ & $\begin{array}{l}30,40,24,15,30,44 \\
30,39,24,17,30,48 \\
30,38,24,15,30,42 \\
30,38,24,14,30,48 \\
30,36,24,15,32,50\end{array}$ & 694801 & $\begin{array}{l}0,0,0,0,0,0 \\
0,0,0,0,0,0 \\
0,0,0,0,0,0 \\
0,0,0,0,0,0 \\
0,0,0,0,0,0\end{array}$ \\
\hline 2 & $\begin{array}{l}30 \\
40 \\
26 \\
30 \\
30 \\
32 \\
\end{array}$ & $\begin{array}{l}170619 \\
144536 \\
144443 \\
145630 \\
144931\end{array}$ & $\begin{array}{l}40,50,32,20,30,60 \\
30,40,32,15,30,48 \\
30,40,32,17,30,46 \\
30,35,32,20,30,50 \\
30,37,32,23,30,48\end{array}$ & 750160 & $\begin{array}{l}0,0,0,0,0,0 \\
0,0,0,0,0,0 \\
0,0,0,0,0,0 \\
0,0,0,0,0,0 \\
0,0,0,0,0,0\end{array}$ \\
\hline 3 & $\begin{array}{l}30 \\
40 \\
18 \\
30 \\
20 \\
32 \\
\end{array}$ & $\begin{array}{l}183044 \\
178905 \\
170547 \\
177920 \\
178959\end{array}$ & $\begin{array}{l}40,50,40,20,40,60 \\
40,50,32,20,40,60 \\
40,50,32,20,30,60 \\
40,50,32,20,40,60 \\
40,55,32,28,30,72\end{array}$ & 889374 & $\begin{array}{l}0,0,0,0,0,0 \\
0,0,0,0,0,0 \\
0,0,0,0,0,0 \\
0,0,0,0,0,0 \\
0,0,0,0,0,0\end{array}$ \\
\hline 4 & $\begin{array}{l}20 \\
30 \\
18 \\
25 \\
20 \\
20\end{array}$ & $\begin{array}{l}209023 \\
199406 \\
198954 \\
199432 \\
200665\end{array}$ & $\begin{array}{l}50,60,40,25,40,72 \\
40,60,40,20,40,72 \\
40,65,40,35,40,72 \\
40,60,44,38,35,72 \\
40,60,40,20,40,64\end{array}$ & 1007481 & $\begin{array}{l}0,0,0,0,0,0 \\
0,0,0,0,0,0 \\
0,0,0,0,0,0 \\
0,0,0,0,0,0 \\
0,0,0,0,0,0\end{array}$ \\
\hline 5 & $\begin{array}{l}20 \\
30 \\
10 \\
25 \\
20 \\
20\end{array}$ & $\begin{array}{l}215198 \\
210139 \\
209519 \\
209863 \\
209379\end{array}$ & $\begin{array}{l}50,60,48,25,40,72 \\
50,60,40,25,40,72 \\
50,65,40,32,40,72 \\
50,60,40,25,40,70 \\
50,55,40,30,40,72\end{array}$ & 1054098 & $\begin{array}{l}0,0,0,0,0,0 \\
0,0,0,0,0,0 \\
0,0,0,0,0,0 \\
0,0,0,0,0,0 \\
0,0,0,0,0,0\end{array}$ \\
\hline 6 & $\begin{array}{l}20 \\
20 \\
10 \\
25 \\
10 \\
8\end{array}$ & $\begin{array}{l}3112742 \\
3114210 \\
3110595 \\
3114297 \\
3111409\end{array}$ & $\begin{array}{l}50,70,48,25,50,84 \\
50,70,48,25,60,78 \\
50,70,60,25,50,80 \\
50,70,50,25,50,82 \\
50,80,48,25,50,84\end{array}$ & 15563254 & $\begin{array}{c}0,0,0,0,0,480000 \\
0,0,0,0,1000000,0 \\
0,0,1600000,0,0,0 \\
0,0,0,0,0,240000 \\
0,2500000,0,0,0,480000\end{array}$ \\
\hline
\end{tabular}

first-echelon supply center to the second-echelon reloading point 1 ; column 20 is the sum of columns $7-12$, that is, the transfer volume of the transfer of the first-echelon supply center to the second-echelon reloading point 2; column 21 is the sum of 13-18, that is, the transfer volume of the first-echelon supply center to the second-echelon reloading point 3.

\section{Result Analysis}

6.1. Different Failure Degree ( $\lambda$ ) Analysis. As shown in Table 8 , change the size and observe the different optimization results of the model and algorithm.

As can be seen from Table 8, with the increasing failure degree $(\lambda)$, the consumption of the whole process is gradually increasing and the demand for spare parts is increasing. As a result, the preset inventory node is difficult to meet the consumption of spare parts during the process of supply.
When $\lambda$ reached $1.4 \times 10^{-4}$, there is a delayed loss, and in the actual supply of spare parts, delay consumption should be eliminated or avoided as far as possible.

6.2. Different Quantity of Inventory to Supply Analysis. As can be seen from Table 9, when the inventory node is reduced, the consumption of spare parts is increasing continuously, the same as the failure degree, and the cost of each period is also increased. On the other hand, the reduction of the inventory node leads to insufficient advance supply time. Eventually, there is a breakdown loss when the inventory node drops to $20,20,10,25,10,8$.

6.3. Compared with Results of Traditional $(s, S)$ Policy. In order to test the effect of the model and algorithm optimization, the model is compared with the traditional $(s, S)$ policy based on different inventory nodes. 
TABle 10: Comparison of No. 6 supply node with the traditional $(s, S)$ policy.

\begin{tabular}{|c|c|c|c|c|}
\hline & Cost of each period (unit: yuan) & Consumption & Total cost (unit: yuan) & Breakdown loss (unit: yuan) \\
\hline \multirow{5}{*}{ Traditional model } & 3078278 & $50,70,48,25,50,84$ & \multirow{5}{*}{15395954} & $0,0,0,0,0,480000$ \\
\hline & 3078580 & $50,70,60,25,60,82$ & & $0,0,160000,0,100000,240000$ \\
\hline & 3079982 & $55,70,52,30,50,82$ & & $0,0,320000,0,0,240000$ \\
\hline & 3079691 & $50,76,48,40,53,80$ & & $0,0,0,0,300000,0$ \\
\hline & 3079422 & $50,70,48,25,48,84$ & & $0,0,0,0,0,480000$ \\
\hline \multirow{5}{*}{ Improved model } & 199304 & $40,60,40,20,40,72$ & \multirow{5}{*}{946741} & $0,0,0,0,0,0$ \\
\hline & 185191 & $40,60,32,20,30,72$ & & $0,0,0,0,0,0$ \\
\hline & 188756 & $40,65,32,20,32,72$ & & $0,0,0,0,0,0$ \\
\hline & 185761 & $40,60,32,25,30,58$ & & $0,0,0,0,0,0$ \\
\hline & 187729 & $38,60,35,20,30,66$ & & $0,0,0,0,0,0$ \\
\hline
\end{tabular}

TABLE 11: Comparison between the improved algorithm and the traditional algorithm.

\begin{tabular}{|c|c|c|c|c|c|}
\hline & & Cost of each period (unit: yuan) & Consumption & Total cost (unit: yuan) & Breakdown loss (unit: yuan) \\
\hline \multirow{5}{*}{ Improved algorithm } & & 137967 & $30,40,24,15,30,44$ & \multirow{5}{*}{694801} & $0,0,0,0,0,0$ \\
\hline & & 139404 & $30,39,24,17,30,48$ & & $0,0,0,0,0,0$ \\
\hline & & 138765 & $30,38,24,15,30,42$ & & $0,0,0,0,0,0$ \\
\hline & & 138758 & $30,38,24,14,30,48$ & & $0,0,0,0,0,0$ \\
\hline & & 139907 & $30,36,24,15,32,50$ & & $0,0,0,0,0,0$ \\
\hline \multirow{15}{*}{ Traditional algorithm } & \multirow{5}{*}{1} & 288765 & $70,40,24,15,30,35$ & \multirow{5}{*}{982756} & $150000,0,0,0,0,0$ \\
\hline & & 279907 & $30,38,24,15,64,58$ & & $0,0,0,0,140000,0$ \\
\hline & & 138569 & $30,37,24,15,30,42$ & & $0,0,0,0,0,0$ \\
\hline & & 137659 & $30,38,25,14,50,48$ & & $0,0,0,0,0,0$ \\
\hline & & 137856 & $30,36,24,15,32,40$ & & $0,0,0,0,0,0$ \\
\hline & \multirow{5}{*}{2} & 186998 & $40,50,40,20,40,60$ & \multirow{5}{*}{867222} & $0,0,0,0,0,0$ \\
\hline & & 160912 & $30,50,32,20,40,60$ & & $0,0,0,0,0,0$ \\
\hline & & 160455 & $30,50,32,20,30,60$ & & $0,0,0,0,0,0$ \\
\hline & & 186751 & $40,50,32,20,40,60$ & & $0,0,0,0,0,0$ \\
\hline & & 172106 & $40,55,32,28,30,72$ & & $0,0,0,0,0,0$ \\
\hline & \multirow{5}{*}{3} & 139967 & $30,37,26,17,30,46$ & \multirow{5}{*}{696718} & $0,0,0,0,0,0$ \\
\hline & & 139204 & $30,36,24,15,30,50$ & & $0,0,0,0,0,0$ \\
\hline & & 138765 & $30,38,24,15,30,42$ & & $0,0,0,0,0,0$ \\
\hline & & 139258 & $30,38,24,15,31,48$ & & $0,0,0,0,0,0$ \\
\hline & & 139524 & $32,39,24,17,30,48$ & & $0,0,0,0,0,0$ \\
\hline
\end{tabular}

Table 10 is the model optimization comparison results based on the inventory node $s_{k}=[60,80,50,45,50,80]$. It can be seen from the table that the consumption of the adjustment model is lower so that the cost of the traditional model is lower than that of the improved model in this paper. However, from the comparison optimized results based on the inventory node $s_{k}=[20,20,10,25,10,8]$ in Table 9, the consumption of the adjustment model proposed in this paper is lower. At the same time, when the inventory node is in this state, the traditional model cannot meet the consumption in the supply period because the consumption of spare parts is not considered. This leads to delays and breakdown loss.

Although the cost of the traditional model is lower than that of this paper when setting lower supply nodes, considering the fault tolerance and stability of the whole model, the model proposed in this paper can meet the lower demand of inventory nodes while ensuring the stable supply of spare parts. It is in order to ensure that there are no delays and delay losses as much as possible and make it more stable and reliable.

6.4. Comparison between Improved Algorithm and Traditional Algorithm. In order to analyze the effect of the improved algorithm, the joint model is also calculated by the traditional PSO algorithm. The comparison result is shown in Table 11.

From Table 11, it is very obvious that the results of the first and second calculation through the traditional algorithm are in the position of local optimization. The system calculates the final result only once. Even the third result is 
higher than the result of improved algorithm. However, with the effect of algorithm error, the final results of them can be seen as the same.

\section{Conclusion}

In this paper, the joint policy combines the inventory policy and spare part supply network. In the joint model, the multiperiod and multiechelon supply network is built, and the $(s, S)$ policy is improved by the random lead time and different customers' maximum inventory. Due to the nonlinear, nonmonotonic, and multiperiodic changes of the established model, an improved PSO algorithm is proposed. The algorithm used in this paper is optimized by adding adaptive inertia weight and penalty function to speed up the optimization efficiency and improve the convergence effect. A case is given. The optional supply scheme is obtained by the proposed algorithm. The sensitivity analysis is used to discuss the influence of important parameters on the model cost. The statistical characteristics of the model are summarized to provide a reference for the next intelligent decision. Except that, the comparison results concluding the traditional $(s, S)$ model and the traditional PSO algorithm are analyzed. We hope that the joint policy and used method can provide a reference for the spare part supply in industry and military.

In this paper, the parameters in the inventory policy are given as known, which can regard as optimized objects in the future research. In addition, it can continue to study the supply of multikind spare parts under different lifetime distribution and so on.

\section{Data Availability}

The data used to support the findings of this study are included within the article.

\section{Conflicts of Interest}

The authors declare that they have no conflicts of interest.

\section{References}

[1] C. C. Sherbrooke, "VARI-METRIC: improved approximations for multi-indenture, multi-echelon availability models," Operations Research, vol. 34, no. 2, pp. 311-319, 1986.

[2] Q. Hu, S. Chakhar, S. Siraj, and A. Labib, "Spare parts classification in industrial manufacturing using the dominancebased rough set approach," European Journal of Operational Research., vol. 262, no. 3, pp. 1136-1163, 2017.

[3] A. A. Ghobbar and C. H. Friend, "Evaluation of forecasting methods for intermittent parts demand in the field of aviation: a predictive model," Computers \& Operations Research., vol. 30, no. 14, pp. 2097-2114, 2003.

[4] R. Min, Q. Chen, and Z. Shen, Spare Parts Supply Science, National Defense Industry Press, 2013.

[5] R. P. Covert and G. C. Philip, "An EOQ model for items with Weibull distribution deterioration," A I I E Transactions., vol. 5, no. 4, pp. 323-326, 1973.

[6] S. Bashyam and M. C. Fu, "Optimization of (s, S) inventory systems with random lead times and a service level constraint,"
Management Science., vol. 44, no. 12-part-2, pp. S243-S256, 1998.

[7] S. Osaki, "An ordering policy with lead time," International Journal of Systems Science., vol. 8, no. 10, pp. 1091-1095, 1977.

[8] M. Issa, A. E. Hassanien, D. Oliva, A. Helmi, I. Ziedan, and A. Alzohairy, "ASCA-PSO: adaptive sine cosine optimization algorithm integrated with particle swarm for pairwise local sequence alignment," Expert Systems with Applications, vol. 99, pp. 56-70, 2018.

[9] C. C. Sherbrooke, "Metric: a multi-echelon technique for recoverable item control," Operations Research, vol. 16, no. 1, pp. 122-141, 1968.

[10] T. S. Vaughan, "Failure replacement and preventive maintenance spare parts ordering policy," European Journal of Operational Research., vol. 161, no. 1, pp. 183-190, 2005.

[11] G. P. Cachon, "Exact evaluation of batch-ordering inventory policies in two-echelon supply chains with periodic review," Operations Research, vol. 49, no. 1, pp. 79-98, 2001.

[12] W. J. Kennedy, J. Wayne Patterson, and L. D. Fredendall, “An overview of recent literature on spare parts inventories," International Journal of Production Economics., vol. 76, no. 2, pp. 201-215, 2002.

[13] U. S. Rao, "Properties of the periodic review $(\mathrm{R}, \mathrm{T})$ inventory control policy for stationary, stochastic demand," M\&SOM., vol. 5, no. 1, pp. 37-53, 2003.

[14] Y. Wang and Q. Shi, "Improved dynamic PSO-based algorithm for critical spare parts supply optimization under ( $\mathrm{T}$, S) inventory policy," IEEE Access., vol. 7, pp. 153694-153709, 2019.

[15] M. C. Reade, A. Delaney, M. J. Bailey et al., "Prospective metaanalysis using individual patient data in intensive care medicine," Intensive Care Medicine, vol. 36, no. 1, pp. 11-21, 2010.

[16] L. Spanjers, J. C. W. van Ommeren, and W. H. M. Zijm, "Closed loop two-echelon repairable item systems," OR Spectrum, vol. 27, no. 2-3, pp. 369-398, 2005.

[17] B.-T. Aharon, G. Boaz, and S. Shimrit, "Robust multi-echelon multi-period inventory control," European Journal of Operational Research., vol. 199, no. 3, pp. 922-935, 2009.

[18] P. K. Aggarwal and K. Moinzadeh, "Order expedition in multiechelon production/distribution systems," IIE Transactions., vol. 26, no. 2, pp. 86-96, 1994.

[19] A. Federgruen and P. Zipkin, "A combined vehicle routing and inventory allocation problem," Operations Research, vol. 32, no. 5, pp. 1019-1037, 1984.

[20] J. Shu, C.-P. Teo, and Z.-J. M. Shen, "Stochastic transportation-inventory network design problem," Operations Research, vol. 53, no. 1, pp. 48-60, 2005.

[21] A. K. Saha, A. Paul, A. Azeem, and S. K. Paul, "Mitigating partial-disruption risk: a joint facility location and inventory model considering customers' preferences and the role of substitute products and backorder offers," Computers \& Operations Research., vol. 117, p. 104884, 2020.

[22] S. Ekinci, D. Izci, and B. Hekimoğlu, "Optimal FOPID speed control of DC motor via opposition-based hybrid Manta ray foraging optimization and simulated annealing algorithm," Arabian Journal for Science and Engineering, vol. 46, no. 2, pp. 1395-1409, 2021.

[23] J. F. Farfán and L. Cea, "Coupling artificial neural networks with the artificial bee colony algorithm for global calibration of hydrological models," Neural Computing and Applications, vol. 33, no. 14, pp. 8479-8494, 2021. 
[24] A. S Sakthivel, A. D Mary, R. Vetrivel, and V. S. Kannan, "Optimal location of SVC for voltage stability enhancement under contingency condition through PSO algorithm," International Journal of Computer Applications, vol. 20, no. 1, pp. 30-36, 2011.

[25] M. Clerc and J. Kennedy, "The particle swarm - explosion, stability, and convergence in a multidimensional complex space," IEEE Trans. Evol. Computat., vol. 6, no. 1, pp. 58-73, 2002.

[26] Wen-Fung Leong and G. G. Yen, "PSO-based multiobjective optimization with dynamic population size and adaptive local archives," IEEE Transactions on Systems, Man, and Cybernetics, Part B (Cybernetics), vol. 38, no. 5, pp. 1270-1293, 2008.

[27] E. Mezura-Montes and C. A. Coello Coello, "An improved diversity mechanism for solving constrained optimization problems using a multimembered evolution strategy," in Genetic and Evolutionary Computation-GECCO 2004, K. Deb, Ed., pp. 700-712, Springer, Berlin Heidelberg, Berlin, Heidelberg, 2004.

[28] Q. Hu, J. E. Boylan, H. Chen, and A. Labib, "OR in spare parts management: a review," European Journal of Operational Research., vol. 266, no. 2, pp. 395-414, 2018.

[29] M. Z. Ruan, Q. M. Li, Y. W. Peng, E. S. Ge, and A. L. Huang, "Model of spare part fill rate for systems of various structures and optimization method," Systems Engineering and Electronics., vol. 33, pp. 1799-1803, 2011.

[30] A. Mahor and S. Rangnekar, "Short term generation scheduling of cascaded hydro electric system using novel self adaptive inertia weight PSO," International Journal of Electrical Power \& Energy Systems., vol. 34, no. 1, pp. 1-9, 2012.

[31] J. Kennedy and R. Eberhart, "Particle swarm optimization," in Proceedings of ICNN'95- International Conference on Neural Networks, IEEE, pp. 1942-1948, Perth, WA, Australia, 1995.

[32] K. Deep, "Madhuri: application of globally adaptive inertia weight PSO to Lennard-Jones problem," Proceedings of the International Conference on Soft Computing for Problem Solving (Soc ProS 2011) December 20-22, 2011, K. Deep, A. Nagar, M. Pant, and J. C. Bansal, Eds., , pp. 31-38, Springer India, India, 2012.

[33] H. Shao and G. Zheng, "Boundedness and convergence of online gradient method with penalty and momentum," Neurocomputing, vol. 74, no. 5, pp. 765-770, 2011.

[34] P. Sincak, "Intelligent technologies-theory and applications: new trends in intelligent technologies," IOS Press, Ohmsha, Amsterdam; Washington, DC: Tokyo, 2002.

[35] A. C. Nearchou, "The effect of various operators on the genetic search for large scheduling problems," International Journal of Production Economics., vol. 88, no. 2, pp. 191-203, 2004. 